\title{
On BMO-regular couples of lattices of measurable functions
}

\author{
by \\ S. V. Kislyakov (St. Petersburg) \\ Dedicated to A. Petczyński on the occasion of his 70th anniversary
}

\begin{abstract}
We introduce a new "weak" BMO-regularity condition for couples $(X, Y)$ of lattices of measurable functions on the circle (Definition 3, Section 9), describe it in terms of the lattice $X^{1 / 2}\left(Y^{\prime}\right)^{1 / 2}$, and prove that this condition still ensures "good" interpolation for the couple $\left(X_{A}, Y_{A}\right)$ of the Hardy-type spaces corresponding to $X$ and $Y$ (Theorem 1, Section 9). Also, we present a neat version of Pisier's approach to interpolation of Hardy-type subspaces (Theorem 2, Section 13). These two main results of the paper are proved in Sections 10-18, where some related material of independent interest is also discussed. Sections 1-8 are devoted to the background and motivations, and also include a short survey of some previously known results concerning BMO-regularity. To a certain extent, the layout of the paper models that of the lecture delivered by the author at the conference in functional analysis in honour of Aleksander Pełczyński (Będlewo, September 22-29, 2002).
\end{abstract}

1. The set-up. By a lattice of measurable functions (or simply a lattice) we mean a quasi-Banach space $X$ consisting of measurable functions on some $\sigma$-finite measure space $(\Sigma, \mu)$ and satisfying the following condition: if $f \in X, g$ is measurable, and $|g| \leq|f|$, then $g \in X$ and $\|g\| \leq C\|f\|$. When talking of a Banach lattice, we also assume that $C=1$. In this paper, for the basic measure space we take the product $(\mathbb{T} \times \Omega, m \times \mu)$, where $m$ is the normalized Lebesgue measure on the unit circle $\mathbb{T}$, and $(\Omega, \mu)$ is some $\sigma$-finite measure space. For technical reasons (see [6]), we often assume that the measure $\mu$ is discrete. This assumption is not too restrictive, however, because still fairly often it can be lifted by approximation by step functions. The case of functions on $\mathbb{T}$ is included by taking a point mass for $\mu$.

2000 Mathematics Subject Classification: 46E25, 46B70, 42B30.

This research has been supported in part by the RFBR, grant 02-01-00267, by the Special Federal Programme "Integration", No. 326.53, and by the grant "Scientific Schools", No. 00-15-96-022. 
Let $N_{+}$be the boundary Smirnov class (see, e.g., [11]). The analytic part $X_{A}$ of a lattice $X$ is defined as follows:

$$
X_{A}=\left\{f \in X: f(\cdot, \omega) \in N_{+} \text {for a.e. } \omega\right\} .
$$

In order to avoid degeneration, we impose the following condition on $X$ : for every nonzero $f$ in $X$ there exists $g \in X$ with $g \geq|f|,\|g\| \leq c\|f\|$, and $\log |g(\cdot, \omega)| \in L^{1}(\mathbb{T})$ for a.e. $\omega$. See $[3,6]$ for more details, in particular, for the proof that a Banach lattice $X$ satisfies this condition if and only if so does its order dual

$$
X^{\prime}=\left\{g: \int|f g| d m d \mu<\infty \text { for all } f \in X\right\} .
$$

In what follows, this condition is tacitly assumed to be satisfied for all lattices that occur.

Clearly, $L^{p}(\mathbb{T})_{A}$ is the Hardy space $H^{p}$ (so, the spaces $X_{A}$ are also called Hardy-type spaces).

2. Interpolation. We recall some facts about interpolation of Hardytype subspaces (for more details see, e.g., the survey [5] and the references therein). If we mean real interpolation, it is most natural to study the basic property of $K$-closedness.

We remind the reader (see [10]) that a subcouple $\left(F_{0}, F_{1}\right)$ of an interpolation couple $\left(E_{0}, E_{1}\right)$ of quasi-Banach spaces is said to be $K$-closed if for every vector $f \in F_{0}+F_{1}$ and every decomposition $f=e_{0}+e_{1}$ with $e_{i} \in E_{i}$ $(i=0,1)$ there exists another decomposition $f=f_{0}+f_{1}$, where $f_{i} \in F_{i}$ and $\left\|f_{i}\right\|_{E_{i}} \leq C\left\|e_{i}\right\|_{E_{i}}(i=0,1)$. Of course, here $C$ must not depend on the particular vectors involved.

If the couple $\left(X_{A}, Y_{A}\right)$ is $K$-closed in $(X, Y)$, we say that the couple $(X, Y)$ is analytically $K$-stable (or $A K$-stable). It is important that this condition is self-dual. To state this in precise terms, we recall that a Banach lattice $X$ of measurable functions has the Fatou property if

$$
f_{n} \rightarrow f \text { a.e., } f_{n} \in X,\left\|f_{n}\right\|_{X} \leq C \Rightarrow f \in X \text { and }\|f\|_{X} \leq C .
$$

It is well known that every order dual $Y^{\prime}$ has this property and that the Fatou property in $X$ is equivalent to the identity $X^{\prime \prime}=X$ (see, e.g., [4]).

Proposition 1. Assume Banach lattices $X$ and $Y$ have the Fatou property. Then the couple $(X, Y)$ is analytically $K$-stable if and only if so is the couple $\left(X^{\prime}, Y^{\prime}\right)$.

If $X$ and $Y$ are $\sigma$-order continuous Banach lattices, then, in a natural way, the Banach duals $X^{*}$ and $Y^{*}$ can be identified with $X^{\prime}$ and $Y^{\prime}$, and in this case Proposition 1 is a well known and easy exercise in separation theorems (moreover, in this case the Fatou property is irrelevant); see 
$[10,5]$. In the present form Proposition 1 is more involved and was proved in $[6$, Lemma 7$]$.

3. BMO-regularity. Analytic $K$-stability occurs fairly often and is rather well understood (see, e.g., [10, 7, 5]). The author's feeling is that in this topic only one serious question remains unanswered, namely, the question about the relationship between this property and some kind of $B M O$-regularity. The latter notion exists in at least two forms: for spaces and for couples.

Definition 1. A quasi-Banach lattice $X$ on $\mathbb{T} \times \Omega$ is said to be $B M O$ regular (see [3]) if for every $f \in X$ with $f \neq 0$ there exists $g \in X$ satisfying $|f| \leq g,\|g\|_{X} \leq C\|f\|_{X}$, and $\|\log g(\cdot, \omega)\|_{\text {BMo }} \leq C$ for a.e. $\omega$, where $C$ does not depend on $f$.

We remind the reader that the BMO-norm of every constant function is 0 , so that the above relations are homogeneous. The function $g$ will be called a BMO-majorant for $f$.

Definition 2. A couple $(X, Y)$ of quasi-Banach lattices on $\mathbb{T} \times \Omega$ is said to be BMO-regular (see [5]) if for every nonzero $x \in X$ and $y \in Y$ there exist $u \in X, v \in Y$ with $|x| \leq u,|y| \leq v,\|u\|_{X} \leq C\|x\|_{X},\|v\|_{Y} \leq C\|y\|_{Y}$, and

$$
\left\|\log \frac{u(\cdot, \omega)}{v(\cdot, \omega)}\right\|_{\mathrm{BMO}} \leq C
$$

for a.e. $\omega$. The pair $(u, v)$ will be called a BMO-majorant for $(x, y)$.

Clearly, if both $X$ and $Y$ are BMO-regular, then the couple $(X, Y)$ is also BMO-regular. The converse is not true. Indeed, a couple of the form $(X, X)$ is always BMO-regular: as a BMO-majorant for a pair $(x, y)$ we can take $(\|x\| \varphi,\|y\| \varphi)$, where $\varphi=x /\|x\| \vee y /\|y\|+\varepsilon \psi, \psi \in X$ is strictly positive a.e., and $\varepsilon$ is a small positive number. On the other hand, not all lattices $X$ are BMO-regular. For example, $L^{p}(w)$ is BMO-regular if and only if $\operatorname{essips}_{\omega \in \Omega}\|\log w(\cdot, \omega)\|<\infty($ see $[3,5])$.

The following fact was proved in [5] (another proof will be given in Subsection 14.2 below).

Proposition 2. Every BMO-regular couple is analytically $K$-stable.

At present no other examples of $A K$-stable couples are known.

4. Duality. In Sections 4-8 we present a summary of known results about BMO-regularity. We can say that for individual spaces this property is fairly well understood, whereas for couples it is still somewhat elusive. 
Since some form of BMO-regularity is conjectured to be equivalent to $A K$-stability, by Proposition 1, BMO-regularity is expected to be a self-dual property. In [6] it was proved that for individual spaces this is really the case.

Proposition $3([6])$. Let $(\Omega, \mu)$ be a discrete space. Suppose a Banach lattice $X$ of measurable functions on $\mathbb{T} \times \Omega$ has the Fatou property. If $X$ is $B M O-r e g u l a r$, then so is $X^{\prime}$.

Since the Fatou property in $X$ is equivalent to the identity $X^{\prime \prime}=X$, the reverse implication is also true.

No analog of this statement for BMO-regular couples is available at present.

5. Arithmetic. For a quasi-Banach lattice $X$ of measurable functions and a positive number $a$, we put

$$
X^{a}=\left\{x:|x|^{1 / a} \in X\right\}, \quad\|x\|_{X^{a}}=\left\||x|^{1 / a}\right\|^{a} .
$$

This is a quasi-Banach lattice, which is Banach if $X$ is Banach and $a \leq 1$.

For two quasi-Banach lattices $X$ and $Y$, we put

$$
X Y=\{x y: x \in X, y \in Y\}, \quad\|f\|_{X Y}=\inf \|x\|_{X}\|y\|_{Y},
$$

where the infimum is taken over all factorizations $f=x y$. The space $X Y$ is a quasi-Banach lattice. If $X$ and $Y$ are Banach lattices and $0<\alpha<1$, then $X^{1-\alpha} Y^{\alpha}$ is also Banach and $\left(X^{1-\alpha} Y^{\alpha}\right)^{\prime}=\left(X^{\prime}\right)^{1-\alpha}\left(Y^{\prime}\right)^{\alpha}$ (see [9]). If $X$ is a Banach lattice, then $X X^{\prime}=L^{1}$ (the Lozanovskiu factorization theorem, see [9]). Next, $\left(L^{\infty}\right)^{a}=L^{\infty}$ for every $a$ and $X L^{\infty}=X$ for every $X$.

The following statements are direct consequences of the definitions.

If $X$ is BMO-regular and $a>0$, then $X^{a}$ is BMO-regular. If both $X$ and $Y$ are BMO-regular, then so is $X Y$.

If the couple $(X, Y)$ is BMO-regular and $a>0$, then the couple $\left(X^{a}, Y^{a}\right)$ is BMO-regular. If the couples $(X, Y)$ and $(E, F)$ are BMO-regular, then so is the couple $(X E, Y F)$.

6. Examples of BMO-regular spaces. Let $\mathcal{H}$ be the harmonic conjugation operator. It acts also on functions on $\mathbb{T} \times \Omega$ in the first variable.

$1^{\circ}$ If $\mathcal{H}$ is bounded on $X$, then $X$ is BMO-regular. See $[5,6]$.

Let $w$ be a positive measurable function on $\mathbb{T} \times \Omega$ (to avoid degeneration, in accordance with Section 1 we must also impose the condition $\log w(\cdot, \omega) \in L^{1}(\mathbb{T})$ for a.e. $\left.\omega\right)$. For $0<p<\infty$, we define $L^{p}(w)$ in the usual way $\left(\|f\|_{L^{p}(w)}=\left(\int|f|^{p} w d m d \mu\right)^{1 / p}\right)$. It is convenient to put $L^{\infty}(w)=\{f$ : $|f| \leq C w$ for some constant $C\}$, with the natural norm.

$2^{\circ}$ For $0<p \leq \infty$, the space $L^{p}(w)$ is BMO-regular if and only if $\operatorname{esssup}_{\omega \in \Omega}\|\log w(\cdot, \omega)\|_{\mathrm{BMO}}<\infty$. 
For $p=\infty$, the "if" part is true because $\|x\| w$ is a BMO-majorant for $x \in L^{\infty}(w)$. Then for $0<p<\infty$ the "if" part is a consequence of $1^{\circ}$ and the arithmetic discussed in Section 5 (note that multiplication of $L^{p}$ by a weighted $L^{\infty}$ leads to a weighted $L^{p}$ ). The "only if" part can be deduced, for example, from Proposition 2 and the results of [7].

$3^{\circ}$ A less trivial example is $L^{\infty}\left(\mathbb{T}, l^{p}\right), 0<p<\infty$ (this can be regarded as a space of functions on $\mathbb{T} \times \mathbb{N}$ ). Here BMO-regularity follows, for instance, from the criterion in Section 7 below.

7. A criterion. With some unessential reservations, a Banach lattice $X$ with the Fatou property is BMO-regular if and only if $\mathcal{H}$ acts on $\left(\left(X^{a}\right)^{\prime}\right)^{1 / 2}$ for some $a>0$.

The "only if" part is an easy consequence of the Helson-Szego" theorem (see [2] concerning the latter). The argument can be found in $[12,5]$. Two proofs of the "if" part are known. One of them is basically due to Rubio de Francia [12] and involves the Grothendieck inequality among other things. See [5] for a detailed exposition. A slight additional restriction on $X$ is needed in that proof, which is certainly satisfied if, e.g., $\mu$ is discrete. In the latter case another proof is available, which is very short modulo the rather involved Proposition 3: by $1^{\circ}$ in Section 6 , the space $\left(\left(X^{a}\right)^{\prime}\right)^{1 / 2}$ is BMO-regular, and it suffices to resort to taking powers and duals.

8. Division. The arithmetic discussed in Section 5 can be supplemented with the following statement.

Proposition 4 (see [6]). Suppose $\mu$ is discrete, $X$ and $Y$ are Banach lattices on $(\mathbb{T} \times \Omega, m \times \mu)$ with the Fatou property, and $0<\alpha<1$. If $X^{1-\alpha} Y^{\alpha}$ and $Y$ are BMO-regular spaces, then so is $X$.

An equivalent formulation is as follows: if $E F$ and $F$ are BMO-regular and $E^{\gamma}, F^{\gamma}$ are Banach lattices for some $\gamma>0$, then $E$ is BMO-regular. That is why we refer to Proposition 4 as the division property.

We include the proof of Proposition 4 because similar patterns will be used below repeatedly. By Proposition 3, $Y^{\prime}$ is BMO-regular, and consequently, so is $\left(X^{1-\alpha} Y^{\alpha}\right)\left(Y^{\prime}\right)^{\alpha}=X^{1-\alpha}\left(L^{1}\right)^{\alpha}$. Passing to the dual again, we see that $\left(X^{1-\alpha}\left(L^{1}\right)^{\alpha}\right)^{\prime}=\left(X^{\prime}\right)^{1-\alpha}\left(L^{\infty}\right)^{\alpha}=\left(X^{\prime}\right)^{1-\alpha}$ is BMO-regular. Then we raise to the power $1 /(1-\alpha)$ and dualize once again.

9. Weak BMO-regularity. For couples, no division theorem is available at present. As will be clear from what follows, such a statement would be equivalent to the conjectural duality theorem. Precisely this gap hinders a handy description of BMO-regular couples. However, this difficulty can be bypassed. To this end, we introduce yet another kind of BMO-regularity. 
Definition 3. A couple $(X, Y)$ of Banach lattices is said to be weakly $B M O-r e g u l a r$ if there is a BMO-regular couple $(E, F)$ of Banach lattices and a number $\alpha>0$ such that the couple $\left(X^{\alpha} E, Y^{\alpha} F\right)$ is BMO-regular.

Basically, this notion serves to substitute the division property. The exponent $\alpha$ is of technical nature. Note that we cannot get rid of $\alpha$ by mere raising to a power because $E^{1 / \alpha}$ and $F^{1 / \alpha}$ may fail to be Banach. However, elimination of $\alpha$ is possible (see the theorem below).

Now we state the first main result of the paper.

Theorem 1. Suppose $(\Omega, \mu)$ is a discrete measure space and $X, Y$ are Banach lattices of measurable functions on $(\mathbb{T} \times \Omega, m \times \mu)$ with the Fatou property.

(a) For the couple $(X, Y)$, weak BMO-regularity still suffices for $A K$ stability.

(b) The following statements are equivalent:

(I) the couple $(X, Y)$ is weakly BMO-regular;

(II) the couple $\left(X L^{1}, Y L^{1}\right)$ is BMO-regular;

(III) the space $X Y^{\prime}$ is BMO-regular.

It is possible to remain within Banach lattices in this statement by taking the power $1 / 2$ throughout in (II) and (III). We still assume the Fatou property in the next corollary.

Corollary. If $\mu$ is discrete, then weak BMO-regularity is a self-dual property.

Proof. Indeed $\left(X^{1 / 2}\left(Y^{\prime}\right)^{1 / 2}\right)^{\prime}=\left(X^{\prime}\right)^{1 / 2} Y^{1 / 2}$, and it suffices to refer to Proposition 3.

I conjecture that, in fact, weak BMO-regularity is the same as BMOregularity for couples.

10. An auxiliary result. First, we discuss statement (b) in Theorem 1. It will be deduced by simple "arithmetic" from the following basic lemma.

Lemma 1. Suppose $\mu$ is a discrete measure, and Banach lattices $X$ and $Y$ have the Fatou property. If the lattice $Y$ and the couple $(X, Y)$ are BMOregular, then the lattice $X$ is BMO-regular.

We prove this statement by using the following result due to N. Kalton [3, Lemma 4.4].

Lemma 2. Let $Z$ be a Banach lattice on $\mathbb{T} \times \Omega$. Suppose there exist constants $c, C$, and $M$ with $0<c<1$ such that for every $f \in X$ with $f \geq 0$, $f \neq 0$, there is a measurable set $A \subset \mathbb{T} \times \Omega$ and a function $g \geq f \chi_{A}$ with the following properties: 
(i) $\left\|f-f \chi_{A}\right\|_{Z} \leq c\|f\|_{Z}$;

(ii) $\|g\|_{Z} \leq M\|f\|_{Z}$;

(iii) $\|\log g(\cdot, \omega)\|_{\mathrm{BMO}} \leq C$ for a.e. $\omega$.

Then $Z$ is a BMO-regular lattice.

Surely, the proof goes by iteration: after the first step the assumption of the lemma is applied to $f-f \chi_{A}$, and so on. A sequence of $g_{i}$ 's satisfying (iii) will arise. The crucial observation is that (iii) can be rephrased with the help of the Helson-Szegö theorem (see, e.g., the monograph [2]): for some $\gamma \leq 1$ depending only on $C$ and for all "reasonable" functions $\varphi$ on $\mathbb{T}$, we have

$$
\int_{\mathbb{T}}|\mathcal{H} \varphi(\cdot)|^{2} g_{i}(\cdot, \omega)^{\gamma} \leq D(C) \int_{\mathbb{T}}|\varphi(\cdot)|^{2} g_{i}(\cdot, \omega)^{\gamma} .
$$

These inequalities can be added, etc. See [3] for the details.

Proof of Lemma 1. By Proposition 4 (note that this is the only point where we use the assumption that $\mu$ is discrete), it suffices to prove that the lattice $X^{1 / 2} Y^{1 / 2}$ is BMO-regular. We verify the conditions of Lemma 2 for $Z=X^{1 / 2} Y^{1 / 2}$. Suppose $0 \leq f \in X^{1 / 2} Y^{1 / 2}$ and $\|f\|=1$. Since $Y$ is BMOregular, by the definition of the norm in $X^{1 / 2} Y^{1 / 2}$ we can find $x \in X$ with $x \geq 0,\|x\|_{X} \leq 1$ and $y \in Y$ with $y \geq 0,\|y\|_{Y} \leq 1$ such that $f \leq D x^{1 / 2} y^{1 / 2}$ and $\|\log y(\cdot, \omega)\|_{\text {BMO }} \leq D$ (here $D$ does not depend on $f$ ). Since the couple $(X, Y)$ is BMO-regular, we can find $x_{1} \geq x$ and $y_{1} \geq y$ such that

$$
\left\|x_{1}\right\|_{X} \leq D_{1}, \quad\left\|y_{1}\right\|_{Y} \leq D_{1}, \quad\left\|\log \frac{x_{1}(\cdot, \omega)}{y_{1}(\cdot, \omega)}\right\|_{\mathrm{BMO}} \leq D_{1} .
$$

(Again, $D_{1}$ does not depend on $f$.) Let $\delta>0$. We put $A=\left\{y / y_{1}>\delta\right\}$ and $g=D \delta^{-1}\left(x_{1} y_{1}^{-1}\right)^{1 / 2} y$, and check that if $\delta$ is sufficiently small, then (i)-(iii) are satisfied with these $g$ and $A$.

First, $g \leq D \delta^{-1} x_{1}^{1 / 2} y^{1 / 2}$, so that $\|g\|_{X^{1 / 2} Y^{1 / 2}} \leq D \delta^{-1} D_{1}^{1 / 2}$ (this is (ii)). Second, clearly, $\|\log g(\cdot, \omega)\|_{\text {BMO }} \leq D_{1} / 2+D$ (this is (iii)). Third,

$$
\left\|f-f \chi_{A}\right\| \leq D\|x\|_{X}^{1 / 2}\left\|y\left(1-\chi_{A}\right)\right\|_{Y}^{1 / 2} \leq D\left\|\delta y_{1}\right\|_{Y}^{1 / 2} \leq D\left(\delta D_{1}\right)^{1 / 2}
$$

(this is (i)). Finally,

$$
\begin{aligned}
g & \geq D \delta^{-1}\left(x_{1} y_{1}^{-1}\right)^{1 / 2} y \chi_{A} \\
& \geq D \delta^{-1}\left(x_{1} y_{1}^{-1}\right)^{1 / 2}\left(\delta y_{1}\right) \chi_{A} \geq D x_{1}^{1 / 2} y_{1}^{1 / 2} \chi_{A} \geq f \chi_{A},
\end{aligned}
$$

as required.

11. Proof of statement (b) in Theorem 1. We start with the implication $(\mathrm{I}) \Rightarrow(\mathrm{III})$. First, assume that the couple $(X, Y)$ itself is BMOregular. Taking the product of two BMO-regular couples $\left(X^{1 / 2}, Y^{1 / 2}\right)$ and $\left(\left(Y^{\prime}\right)^{1 / 2},\left(Y^{\prime}\right)^{1 / 2}\right)$ and recalling the Lozanovskiı factorization theorem, we see 
that the couple $\left(X^{1 / 2}\left(Y^{\prime}\right)^{1 / 2},\left(L^{1}\right)^{1 / 2}\right)=\left(X^{1 / 2}\left(Y^{\prime}\right)^{1 / 2}, L^{2}\right)$ is BMO-regular. Since $L^{2}$ is BMO-regular, (III) follows from Lemma 1.

In the general case, we only know that the couple $\left(X^{a} E, Y^{a} F\right)$ is BMOregular for some $a>0$ and some BMO-regular couple $(E, F)$ of Banach lattices. We raise the former couple to the power $\gamma=(a+1)^{-1}$ to obtain the BMO-regularity of the couple $\left(X^{1-\gamma} E^{\gamma}, Y^{1-\gamma} F^{\gamma}\right)$, in which both elements are Banach. By the first part of the proof, the spaces $\left(X^{1-\gamma} E^{\gamma}\right)\left(Y^{1-\gamma} F^{\gamma}\right)^{\prime}=$ $\left(X Y^{\prime}\right)^{1-\gamma}\left(E F^{\prime}\right)^{\gamma}$ and $E F^{\prime}$ are BMO-regular. Application of Proposition 4 (see also the comments after it) shows that $X Y^{\prime}$ is BMO-regular, as required.

Now we prove that (III) $\Rightarrow$ (II). For this we observe that the couple $\left(X Y^{\prime}, L^{1}\right)$ is BMO-regular, multiply it by the BMO-regular couple $(Y, Y)$, and again use the fact that $Y Y^{\prime}=L^{1}$.

Finally, the implication (II) $\Rightarrow(\mathrm{I})$ is trivial.

12. Strong $A K$-stability. Before discussing statement (a) of Theorem 1 , we make a technical remark. The $K$-closedness of the couple $\left(X_{A}, Y_{A}\right)$ in $(X, Y)$ means that an arbitrary measurable decomposition of a function $f \in X_{A}+Y_{A}$ can be replaced by an "analytic" decomposition with good estimates of norms. It is natural to ask whether the same can be done under the assumption $f \in(X+Y)_{A}$ instead of $f \in X_{A}+Y_{A}$. If yes, we say that the couple $(X, Y)$ is strongly $A K$-stable. Often this property is satisfied automatically in the presence of $A K$-stability.

Lemma 3. (a) If $X$ and $Y$ are Banach lattices having the Fatou property and the couple $(X, Y)$ is AK-stable, then it is strongly AK-stable.

(b) If $(X, Y)$ is a BMO-regular couple of quasi-Banach lattices, then it is strongly AK-stable.

Since we are going to reprove Proposition 2 anyway, we postpone the explanation of (b) till that moment. Now, we prove (a). Suppose $f(\cdot, \omega) \in$ $N_{+}$for a.e. $\omega$ and $|f| \leq g+h$, where $g \in X, h \in Y$. Without loss of generality we assume that $\log g(\cdot, \omega) \in L^{1}(\mathbb{T})$ for a.e. $\omega$. For every $n \in \mathbb{N}$ we construct the "outer" function

$$
\varphi_{n}=\exp \left[\log \left(1 \wedge \frac{n g}{|f|}\right)+i \mathcal{H}\left(\log \left(1 \wedge \frac{n g}{|f|}\right)\right)\right] .
$$

Then $\left|f \varphi_{n}\right| \leq n g$, so that $f \varphi_{n} \in X_{A} \subset X_{A}+Y_{A}$. Next, $\left|\varphi_{n}\right| \leq 1$, whence $\left|f \varphi_{n}\right| \leq g+h$. By $K$-closedness, $f \varphi_{n}=g_{n}+h_{n}$, where $g_{n} \in X_{A}$, $h_{n} \in Y_{A},\left\|g_{n}\right\|_{X} \leq C\|g\|_{X}$, and $\left\|h_{n}\right\|_{Y} \leq C\|h\|_{Y}$.

Now, $\varphi_{n} \rightarrow 1$ in measure on every set of finite measure, whence $f \varphi_{n} \rightarrow f$ in the same sense. Passing to a subsequence, we can make this convergence fast. We use the (nontrivial) result of Bukhvalov and Lozanovskiu $[4$, Chapter $10, \S 5]$ stating that the Fatou property implies that some "simultaneous" finite convex combinations of the form $G_{k}=\sum_{n \geq k} \lambda_{n}^{(k)} g_{n}$ and 
$H_{k}=\sum_{n>k} \lambda_{n}^{(k)} g_{n}$ converge (say, to $u$ and $v$ ) in the same sense as $k \rightarrow \infty$. It can easily be arranged that the same convex combinations of $f \varphi_{n}$ 's converge to $f$. Finally, by the Fatou property, the balls of $X_{A}$ and $Y_{A}$ are closed under convergence in measure on every set of finite measure [6, Lemma 3]. Thus, $u \in X_{A}, v \in Y_{A}$, and $f=u+v$ with the required norm estimates.

13. A version of Pisier's method. In [10] G. Pisier proposed a method for verifying $A K$-stability. See also the survey [5] about this. The original version of that method was based on duality (as in Proposition 1) and a construction involving powers of the lattices in question. Here we give a neat version of this second ingredient, which is another main result of the paper.

Theorem 2. Suppose Banach lattices $X, Y$, and $F$ have the Fatou property. If for some $\alpha \in(0,1)$ the couple $\left(F^{1-\alpha} X^{\alpha}, F^{1-\alpha} Y^{\alpha}\right)$ is analytically $K$-stable, then so is the couple $(X, Y)$.

We shall apply this theorem only with $F=L^{\infty}$. Then it reads as follows: if $\left(X^{\alpha}, Y^{\alpha}\right)$ is $A K$-stable for some $\alpha \in(0,1)$, then $(X, Y)$ is $A K$-stable. This differs from Pisier's original version [10] and some subsequent refinements (see, e.g., [5]) by the absence of any additional "interpolation" assumptions about the spaces in question.

14. Applications. We postpone the proof of Theorem 2 to give some applications of it.

14.1. First, we prove statement (a) of Theorem 1. By statement (b) of that theorem, the couple $\left(X^{1 / 2}\left(L^{1}\right)^{1 / 2}, Y^{1 / 2}\left(L^{1}\right)^{1 / 2}\right)$ is BMO-regular, and Theorem 2 applies. However, we may get the result by using only the version of Theorem 2 with $F=L^{\infty}$, as follows. By Proposition 2, the above couple is $A K$-stable, and by Proposition 1 so is the couple of the order duals, i.e., $\left(\left(X^{\prime}\right)^{1 / 2},\left(Y^{\prime}\right)^{1 / 2}\right)$. By the restricted version of Theorem 2, the couple $\left(X^{\prime}, Y^{\prime}\right)$ is $A K$-stable, and another reference to Proposition 1 finishes the proof.

14.2. Now we prove Proposition 2 ; moreover, we verify statement (b) of Lemma 3. The beginning is as in [5]. Let $(X, Y)$ be a BMO-regular couple of quasi-Banach lattices, let $f \in(X+Y)_{A}$, and let $|f| \leq g+h$, where $g \in X, h \in Y$. We take a BMO-majorant (see Definition 2) $(u, v)$ for $(x, y)$. Clearly, $f \in\left(L^{\infty}(u)+L^{\infty}(v)\right)_{A}$, and a moment's reflection shows that it suffices to prove that the couple $\left(L^{\infty}(u), L^{\infty}(v)\right)$ is strongly $A K$-stable. By statement (a) of Lemma 3, this will be proved if we show that this couple is $A K$-stable. A change of density reduces this to the $A K$-stability of the couple $\left(L^{\infty}(w), L^{\infty}\right)$, where $w=u / v$, so that $\|\log w(\cdot, \omega)\|_{\text {BMO }} \leq C$ for a.e. $\omega$. 
Now, in contradistinction to the procedures in [5], we use Pisier's method. Theorem 2 shows that, instead of $\left(L^{\infty}(w), L^{\infty}\right)$, we may consider the couple $\left(L^{\infty}(w)^{\alpha},\left(L^{\infty}\right)^{\alpha}\right)=\left(L^{\infty}\left(w^{\alpha}\right), L^{\infty}\right)$ for small $\alpha>0$. By duality (Proposition 1$)$, it suffices to prove that the couple $\left(L^{1}\left(w^{\alpha}\right), L^{1}\right)$ is $A K$-stable (note that $L^{\infty}(a)^{\prime}=L^{1}(a)$ for every weight $\left.a\right)$. Another application of Theorem 2 reduces the matter to the couple $\left(L^{1}\left(w^{\alpha}\right)^{1 / 2},\left(L^{1}\right)^{1 / 2}\right)=\left(L^{2}\left(w^{\alpha}\right), L^{2}\right)$.

But if $\alpha$ is small, then the function $w(\cdot, \omega)^{\alpha}$ satisfies the Helson-Szegö condition (see [2]) uniformly in $\omega$, i.e., the Riesz projection (in the first variable) is bounded on $L^{2}\left(w^{\alpha}\right)$. Since it is bounded also on $L^{2}$, the result follows.

15. Multiplication and division. The proof of Theorem 2 presented below is almost entirely "soft": the principal ingredient is the statement about abstract interpolation couples presented in the next section. Here we discuss another ingredient, related to the specific features of the spaces involved.

LEMMA 4. Let $X, Y$, and $F$ be quasi-Banach lattices of measurable functions on $(\mathbb{T} \times \Omega, m \times \mu)$. If the couple $(X, Y)$ is strongly AK-stable, then so is the couple $(X F, Y F)$.

Proof. Let $f \in(X F+Y F)_{A}$, and let $|f| \leq g+h$, where $g \in X F, h \in Y F$. Then $g=x e_{1}, h=y e_{2}$, where $\left\|e_{1}\right\|_{F}=\left\|e_{2}\right\|_{F}=1,\|x\|_{X} \leq 2\|g\|_{X F}$, $\|y\|_{Y} \leq 2\|h\|_{Y F}$. We put $a=e_{1} \vee e_{2}$ and find $b \geq a$ such that $\|b\|_{F} \leq C\|a\|_{F}$ and $\log a(\cdot, \omega) \in L^{1}(\mathbb{T})$ for a.e. $\omega$.

Next, let $B=\exp (\log b+i \mathcal{H}(\log b))$. Then $\left|f B^{-1}\right| \leq x+y$, so that $f B^{-1} \in(X+Y)_{A}$. By assumption, we can write $f B^{-1}=x_{1}+y_{1}$, where $x_{1} \in X_{A}, y_{1} \in Y_{A},\left\|x_{1}\right\|_{X} \leq C\|x\|_{X}$, and $\left\|y_{1}\right\|_{Y} \leq C\|y\|_{Y}$. Then $f=$ $B x_{1}+B y_{1}$ is the required decomposition of $f$.

Corollary. If $X, Y$, and $F$ are Banach lattices with the Fatou property and $0<\alpha<1$, then the couples $\left(F^{1-\alpha} X^{\alpha}, F^{1-\alpha} Y^{\alpha}\right)$ and $\left(X^{\alpha}, Y^{\alpha}\right)$ may be (strongly) AK-stable only simultaneously.

Proof. Under the assumptions of the corollary, strong $A K$-stability is the same as $A K$-stability.

If the couple $\left(F^{1-\alpha} X^{\alpha}, F^{1-\alpha} Y^{\alpha}\right)$ is strongly $A K$-stable, then by Lemma 4 so is the couple $\left(\left(F^{\prime} F\right)^{1-\alpha} X^{\alpha},\left(F^{\prime} F\right)^{1-\alpha} Y^{\alpha}\right)=\left(\left(L^{1}\right)^{1-\alpha} X^{\alpha}\right.$, $\left.\left(L^{1}\right)^{1-\alpha} Y^{\alpha}\right)$. Passing to the duals (Proposition 1$)$, we see that the couple $\left(\left(X^{\prime}\right)^{\alpha},\left(Y^{\prime}\right)^{\alpha}\right)$ is $A K$-stable. Next, we multiply it by $\left(L^{1}\right)^{1-\alpha}$ and again pass to the duals.

In the other direction we simply multiply the couple $\left(X^{\alpha}, Y^{\alpha}\right)$ by $F^{1-\alpha}$ in accordance with Lemma 4. 
16. Gluing scales. A somewhat weaker version of the proposition below was proved in [8] (see also the survey [5]). Let $\left(X_{0}, X_{1}\right)$ be an interpolation couple of quasi-Banach spaces, and let $\theta \in(0,1)$. We recall that a space $E$ compatible with the couple $\left(X_{0}, X_{1}\right)$ is said to be of class $\mathcal{C}\left(\theta, X_{0}, X_{1}\right)$ (see $\left[1\right.$, pp. 48, 56]) if $E \subset\left(X_{0}, X_{1}\right)_{\theta, \infty}$ (continuous inclusion) and $\|e\|_{E} \leq C\|e\|_{X_{0}}^{1-\theta}\|e\|_{X_{1}}^{\theta}$ for $e \in X_{0} \cap X_{1}$.

Proposition 5. Let $\left(X_{0}, X_{1}\right)$ be an interpolation couple of quasi-Banach spaces, and let $Y_{0}$ and $Y_{1}$ be closed subspaces of $X_{0}$ and $X_{1}$, respectively. Suppose $0<\theta<\delta<1$, and suppose $E_{0} \in \mathcal{C}\left(\theta, X_{0}, X_{1}\right), E_{1} \in \mathcal{C}\left(\delta, X_{0}, X_{1}\right)$. Let $F_{0}$ and $F_{1}$ be closed subspaces of $E_{0}$ and $E_{1}$ both containing $Y_{0} \cap Y_{1}$ and satisfying $F_{0} \subset Y_{0}+F_{1}, F_{1} \subset F_{0}+Y_{1}$. If the couples $\left(Y_{0}, F_{1}\right)$ and $\left(F_{0}, Y_{1}\right)$ are $K$-closed in $\left(X_{0}, E_{1}\right)$ and $\left(E_{0}, X_{1}\right)$, respectively, then the couple $\left(Y_{0}, Y_{1}\right)$ is $K$-closed in $\left(X_{0}, X_{1}\right)$.

Vaguely, the proposition says that if $K$-closedness occurs on two "overlapping intervals" $(0, \delta)$ and $(\theta, 1)$ of the "real interpolation scale", we can "glue" these intervals to obtain $K$-closedness throughout.

Proof. In [8] (and in [5]) this fact was proved under the following stronger assumptions:

$$
\begin{array}{ll}
E_{0}=\left(X_{0}, X_{1}\right)_{\theta, p}, & E_{1}=\left(X_{0}, X_{1}\right)_{\delta, q}, \\
F_{0}=\left(Y_{0}, Y_{1}\right)_{\theta, p}, & F_{1}=\left(X_{0}, X_{1}\right)_{\delta, q}
\end{array}
$$

for some $p, q \in(0, \infty]$. If we show that, in Proposition $5, F_{0}$ is of class $\mathcal{C}\left(\theta, X_{0}, X_{1}\right)$ and $F_{1}$ is of class $\mathcal{C}\left(\delta, Y_{0}, Y_{1}\right)$, the statement can easily be reduced to that particular case by the reiteration theorem and the Holmstedt formula (see [1, Theorems 3.5.3 and 3.11.5, and Corollary 3.6.2]; the initial $\theta$ and $\delta$ change slightly after this).

We only prove that $F_{0} \in \mathcal{C}\left(\theta, X_{0}, X_{1}\right)$. (The relation $F_{1} \in \mathcal{C}\left(\delta, Y_{0}, Y_{1}\right)$ is verified in a similar way.) Since $E_{0} \in \mathcal{C}\left(\theta, X_{0}, X_{1}\right)$, the inequality $\|a\|_{F_{0}} \leq$ $C\|a\|_{Y_{0}}^{1-\theta}\|a\|_{Y_{1}}^{\theta}, a \in Y_{0} \cap Y_{1}$, is clear. It remains to show that $F_{0}$ is included continuously in $\left(Y_{0}, Y_{1}\right)_{\theta, \infty}$.

By the reiteration theorem, we have

$$
\begin{array}{ll}
E_{0} \subset\left(X_{0}, X_{1}\right)_{\theta, \infty}=\left(X_{0}, E_{1}\right)_{\eta, \infty}, & \text { where } \eta=\theta \delta^{-1}, \\
E_{1} \subset\left(X_{0}, X_{1}\right)_{\delta, \infty}=\left(E_{0}, X_{1}\right)_{\xi, \infty}, & \text { where } \delta=(1-\xi) \theta+\xi .
\end{array}
$$

Suppose $f \in F_{0}$ and $\|f\|_{F_{0}}=1$. By (1), for every $t>0$ we can write $f=u+v$, where $u \in X_{0}, v \in E_{1},\|u\|_{X_{0}} \leq C t^{\eta}$, and $\|v\|_{E_{1}} \leq C t^{\eta-1}$. By $K$-closedness, we may assume that $u \in Y_{0}$ and $v \in F_{1}$ (surely, $C$ changes, but it remains independent of $f$ and $t$ ).

We do the same for $v$ using (2): for every $\tau>0$, we can write $v=f^{\prime}+w$, where $f^{\prime} \in F_{0}, w \in Y_{1},\left\|f^{\prime}\right\|_{F_{0}} \leq C^{\prime} \tau^{\xi} t^{\eta-1}$, and $\|w\|_{Y_{1}} \leq C^{\prime} \tau^{\xi-1} t^{\eta-1}$. This leads to the relation $f=(u+w)+f^{\prime}$. 
Now, we introduce a new parameter $s>0$ and fix $t$ and $\tau$ so as to have

$$
C t^{\eta}=A s^{\theta}, \quad C^{\prime} \tau^{\xi-1} t^{\eta-1}=A s^{\theta-1},
$$

where $A$ is a constant to be chosen momentarily. Simple calculations show that $\left\|f^{\prime}\right\|_{F_{0}} \leq C^{\prime \prime} A^{-\gamma}$, where $\gamma>0$ depends only on $\theta$ and $\delta$. Taking $A$ in such a way that $C^{\prime \prime} A^{-\gamma}=1 / 2$, for the decomposition $f=u+w+f^{\prime}$ we obtain $\|u\|_{Y_{0}} \leq A s^{\theta},\|w\|_{Y_{1}} \leq A s^{\theta-1}$, and $\left\|f^{\prime}\right\|_{F_{0}} \leq \frac{1}{2}$. The error term $f^{\prime}$ is eliminated by iteration: the same procedure yields $f^{\prime}=u_{1}+v_{1}+f^{\prime \prime}$, where $\left\|u_{1}\right\|_{Y_{0}} \leq \frac{1}{2} A s^{\theta},\left\|w_{1}\right\|_{Y_{1}} \leq \frac{1}{2} A s^{\theta-1},\left\|f^{\prime \prime}\right\|_{F_{0}} \leq \frac{1}{4}$, and so on.

17. Proof of Theorem 2. By the Corollary to Lemma 4, we may assume that the couple $\left(X^{\alpha}, Y^{\alpha}\right)$ is (strongly) $A K$-stable. Next, we may assume that $\alpha>1 / 2$ (otherwise we repeat the argument several times). In this case we prove a slightly stronger statement.

Lemma 5. Let $1 / 2<\alpha<1$, and let $U$ and $V$ be quasi-Banach lattices. If the couple $\left(U^{\alpha}, V^{\alpha}\right)$ is strongly AK-stable, then the couple $(U, V)$ is $A K$ stable.

Observe that, under the conditions of Theorem 2, strong $A K$-stability arises automatically by the Fatou property (Lemma 3).

Proof of Lemma 5. By Lemma 4, the couples $\left(U^{1-\alpha} U^{\alpha}, U^{1-\alpha} V^{\alpha}\right)=$ $\left(U, U^{1-\alpha} V^{\alpha}\right)$ and $\left(U^{\alpha} V^{1-\alpha}, V^{\alpha} V^{1-\alpha}\right)=\left(U^{1-(1-\alpha)} V^{1-\alpha}, V\right)$ are strongly $A K$-stable. We verify the conditions of Proposition 5 with $\theta=1-\alpha, \eta=\alpha$ (note that $\theta<\eta$ because $\alpha>1 / 2$ ), $X_{0}=U, X_{1}=V, Y_{0}=U_{A}, Y_{1}=$ $V_{A}$ and $E_{0}=U^{1-\theta} V^{\theta}, E_{1}=U^{1-\delta} V^{\delta}, F_{0}=\left(E_{0}\right)_{A}, F_{1}=\left(E_{1}\right)_{A}$. Indeed, the Young inequality says that $\left|x^{1-\theta} y^{\theta}\right| \leq t^{\theta}(1-\theta)|x|+t^{\theta-1} \theta|y|$, whence $E_{0} \subset\left(X_{0}, X_{1}\right)_{\theta, \infty}$. Next, clearly, $\left\|x^{1-\theta} y^{\theta}\right\|_{E_{0}} \leq\|x\|_{X_{0}}^{1-\theta}\|y\|_{X_{1}}^{\theta}$, so that $E_{0} \in$ $\mathcal{C}\left(\theta, X_{0}, X_{1}\right)$. Similarly, $E_{1} \in \mathcal{C}\left(\delta, X_{0}, X_{1}\right)$. The inclusion $Y_{0} \cap Y_{1} \subset F_{0} \cap F_{1}$ is obvious, and the inclusions $F_{0} \subset Y_{0}+F_{1}$ and $F_{1} \subset F_{0}+Y_{1}$ follow from strong $A K$-stability and the inclusions $E_{0} \subset X_{0}+E_{1}, E_{1} \subset E_{0}+X_{1}$.

18. Our knowledge concerning the question of whether $A K$-stability implies some form of BMO-regularity is still incomplete. Beyond the positive answer for weighted $L^{p}$-spaces (see [7]), I can only name Theorem 1 in [6], which requires one supplementary variable.

On the measure space $\mathbb{Z}$ with the counting measure, consider the weight $w_{\lambda}$ given by $w_{\lambda}(n)=\lambda^{n}(\lambda>1)$. If $X$ is a lattice on $\mathbb{T} \times \Omega$, then the symbols like $X\left(l^{p}(\mathbb{Z})\right)=X\left(l^{p}\right)$ and $X\left(l^{p}\left(w_{\lambda}\right)\right)$ have an obvious meaning.

Theorem 1 in [6] says that if $\mu$ is discrete and $X$ is a Banach lattice on $(\mathbb{T} \times \Omega, m \times \mu)$ having the Fatou property, then $X$ is BMO-regular if and only if the couple $\left(X\left(l^{r}\right), L^{\infty}\left(l^{\infty}\left(w_{\lambda}\right)\right)\right.$ is $A K$-stable for some (equivalently, for all) $r \in[1, \infty)$ and $\lambda>1$. By duality, the same is true if we replace 
$L^{\infty}\left(l^{\infty}\left(w_{\lambda}\right)\right)$ by $L^{1}\left(l^{1}\left(w_{\lambda}\right)\right)$ and the condition $r \in[1, \infty)$ by $r \in(1, \infty]$. The results of the present paper allow us to supplement these statements. We present two facts as examples. Other exercises in the same spirit are possible.

COROLlary 1. Under the above assumptions, if the couple $\left(X^{1 / p}\left(l^{r}\right)\right.$, $L^{p}\left(l^{p}\left(w_{\lambda}\right)\right)$ is $A K$-stable for some $\lambda>1$ and $r \in(p, \infty]$, then $X$ is BMOregular.

Corollary 2. If $Y$ is another Banach lattice on $\mathbb{T} \times \Omega$, the couple $\left(X\left(l^{p}\right), Y\left(l^{1}\left(w_{\lambda}\right)\right)\right.$ is $A K$-stable for some $\lambda>1$ and some $p \in(1, \infty]$, and $X Y^{\prime}$ is a Banach lattice, then $X Y^{\prime}$ is BMO-regular. The converse is also true (for all $\lambda>1$ and $p \in(1, \infty])$, even without the assumption that $X Y^{\prime}$ is a Banach lattice.

Corollary 1 is quite easy. We indicate the proof of Corollary 2. For the direct statement, we multiply the couple mentioned in the corollary by $Y^{\prime}\left(l^{\infty}\right)$ to obtain the $A K$-stability of the couple $\left(\left(X Y^{\prime}\right)\left(l^{p}\right), L^{1}\left(l^{1}\left(w_{\lambda}\right)\right)\right.$, and refer to the preceding discussion. For the converse, we show that the couple mentioned in the corollary is weakly BMO-regular. By (b)(III) in Theorem 1, it suffices to show that the space $\left(X Y^{\prime}\right)\left(l^{p}\left(w_{\lambda^{p}}\right)\right)$ is BMO-regular, and this can be done as in the proof of the corollary in Subsection 2.1 of [6].

\section{References}

[1] J. Bergh and J. Löfström, Interpolation Spaces, Grundlehren Math. Wiss. 223, Springer, Berlin, 1976.

[2] J. B. Garnett, Bounded Analytic Functions, Academic Press, New York, 1981.

[3] N. J. Kalton, Complex interpolation of Hardy-type subspaces, Math. Nachr. 171 (1995), 227-258.

[4] L. V. Kantorovich and G. P. Akilov, Functional Analysis, 2nd ed., Nauka, Moscow, 1977 (in Russian); English transl.: Pergamon Press, Oxford, New York, 1982.

[5] S. V. Kislyakov, Interpolation of $H^{p}$-spaces: some recent developments, in: Function Spaces, Interpolation Spaces, and Related Topics (Haifa, 1995), Israel Math. Conf. Proc. 13, Bar-Ilan Univ., Ramat Gan, 1999, 102-140.

[6] - On BMO-regular lattices of measurable functions, Algebra i Analiz 14 (2002), no. 2, 117-135 (in Russian); English transl.: St. Petersburg Math. J. 14 (2003), no. 2 .

[7] S. V. Kislyakov and Q. H. Xu, Interpolation of weighted and vector-valued Hardy spaces, Trans. Amer. Math. Soc. 343 (1994), 1-34.

[8] —, -, Real interpolation and singular integrals, Algebra i Analiz 8 (1996), no. 4, 75109 (in Russian); English transl.: St. Petersburg Math. J. 8 (1997), no. 4, 593-615.

[9] G. Ya. Lozanovskiǔ, Certain Banach lattices, Sibirsk. Mat. Zh. 10 (1969), 584-594 (in Russian); English transl.: Siberian Math. J. 10 (1969), 419-431.

[10] G. Pisier, Interpolation between $H^{p}$ spaces and noncommutative generalizations. I, Pacific J. Math. 155 (1992), 341-368.

[11] I. I. Privalov, Boundary Properties of Analytic Functions, Gosud. Izdat. Tekhn.Teor. Lit., Moscow, 1950 (in Russian). 
[12] J. L. Rubio de Francia, Linear operators in Banach lattices and weighted $L^{2}$ inequalities, Math. Nachr. 133 (1987), 197-209.

Russian Academy of Sciences

Steklov Mathematical Institute

St. Petersburg Department

Fontanka 27

St. Petersburg 191023, Russia

E-mail: skis@pdmi.ras.ru

Received November 18, 2002

Revised version April 10, 2003

$(5080)$ 